\title{
Input-to-State Stability for Curve Tracking Control: A Constructive Approach
}

Michael Malisoff
Frédéric Mazenc
Fumin Zhang

\begin{abstract}
We analyze an important class of feedback controllers for curve tracking problems for robotics. Earlier experimental work suggested the robust performance of the control laws under perturbations. In this note, we use input-tostate stability to prove predictable tolerance and safety bounds that guarantee robust performance. Our work uses an invariant polygon argument and a new strict Lyapunov function design. We demonstrate our findings in simulations.

Key Words-Curve tracking, robotics, robustness
\end{abstract}

\section{INTRODUCTION}

Curve tracking plays a key role in control and path planning for mobile robots. The work [5] showed that if a mobile robot can follow boundaries of obstacles in a two dimensional environment, then simple path planning "bug" algorithms can make the robot navigate to any accessible location from any initial position. Subsequent work [7] provided generic feedback control laws that achieve autonomous curve tracking for curves that are either an obstacle boundary or a desired smooth path. The original work [7] was for wheeled mobile robots and was based on the "unicycle model". The interaction between a unit speed mobile robot and its projection on a curve can be modeled by two nonlinear equations, one for the distance, and the other for the bearing between the robot and the curve, namely,

$$
\left\{\begin{array}{l}
\dot{\rho}=-\sin \phi \\
\dot{\phi}=\frac{\kappa \cos \phi}{1+\kappa \rho}-u_{2}
\end{array}\right.
$$

where $\rho$ is the relative distance, $\phi$ is the bearing, $\kappa$ is the (positive) curvature at the closest point, $u_{2}$ is the steering control, and the state space is $\mathscr{X}=(0,+\infty) \times(-\pi / 2, \pi / 2)$. Using a nonstrict Lyapunov function, one can design a feedback control law to achieve asymptotic stabilization of an equilibrium state corresponding to constant distance $\left(\rho=\rho_{0}\right)$ and zero bearing $(\phi=0)$, which occurs when the robot moves parallel to the curve [13]. Later generalizations covered chain form systems [9] that capture the nonholonomic kinematics of a large scope of mobile robots, including a kinematic car with trailers. This led to extended curve tracking control laws.

Malisoff is with Department of Mathematics, Louisiana State University, 303 Lockett Hall, Baton Rouge, LA 70803-4918, malisoff@1su.edu.

Mazenc is with Team INRIA DISCO, CNRS-Supélec, 3 rue Joliot Curie, 91192, Gif-sur-Yvette, France, Frederic.MAZENC@1ss.supelec.fr.

Zhang is with the School of Electrical and Computer Engineering, Georgia Institute of Technology, 210 Technology Circle, Savannah, GA 31322, fumin@gatech.edu.

The first author was supported by AFOSR Grant FA9550-09-1-0400 and NSF Grant ECCS-1056255. The third author was supported by ONR Grants N00014-08-1-1007 and N00014-09-1-1074, and NSF Grants ECCS0841195, ECCS-0845333 (CAREER), ECCS-1056253, and CNS-0931576.
There were several recent improvements for curve tracking control [8]. For example, [2] reformulated the two dimensional model (1) using Lie group theory and gave a natural extension to curve tracking in three dimensional space. Curve tracking controllers also serve as foundations for cooperative controllers that track motion patterns for multiple mobile robots, including applications to ocean sensing [12], [14]. The control laws have been tested in other real life situations, including farming [4], obstacle avoidance in corridors [15], and ocean sampling [12], where they have been shown to give reliable performance under severe perturbations in $\rho$, $\phi, \kappa$, and $u_{2}$. Such experiments strongly suggest that the curve tracking control laws are robust, so it is worthwhile to justify the robustness using analytical tools. This theoretical understanding is important for giving predictable tolerance and safety bounds for the curve tracking control laws that are used in mobile robot applications.

Input-to-state stability (ISS) was introduced in [10] and is a powerful framework for studying robustness of nonlinear controllers under actuator errors; see [11] for a survey. Actuator errors naturally arise from uncertainty in controller implementations, and can be modeled as additive perturbations on the controllers. It is natural to use ISS in robotics, to obtain a quantitative measure of the performance of the curve tracking. Certain curve tracking control laws for chained form systems [8] lead to linear closed loop dynamics, but the linear dynamics lack the required invariance properties for the restricted state space $\mathscr{X}$. To see how, note that if we take the new state variable $z=\sin \phi$, then (1) becomes

$$
\left\{\begin{aligned}
\dot{\rho} & =-z \\
\dot{z} & =\frac{\kappa\left(1-z^{2}\right)}{1+\kappa \rho}-\sqrt{1-z^{2}} u_{2} .
\end{aligned}\right.
$$

Given any constant positive control gains $K_{1}$ and $K_{2}$, the feedback linearizing control law

$$
u_{2}=\frac{1}{\sqrt{1-z^{2}}}\left\{\frac{\kappa\left(1-z^{2}\right)}{1+\kappa \rho}-K_{1}\left(\rho-\rho_{0}\right)+K_{2} z\right\}
$$

gives the linear closed loop dynamics

$$
\left\{\begin{aligned}
\dot{\rho} & =-z \\
\dot{z} & =K_{1}\left(\rho-\rho_{0}\right)-K_{2} z
\end{aligned}\right.
$$

Since (4) is a linear system in $\left(\rho-\rho_{0}, \phi\right)$, exponential stability of the equilibrium requires no more than asymptotic stability, and such exponential stability ensures ISS for linear systems [3]. However, there is no possible choice of the constants $K_{i}$ 's such that $\mathscr{X}_{\text {new }}=(0,+\infty) \times(-1,1)$ is forwardly invariant for (4). In fact, if we take any constant vector $\left(K_{1}, K_{2}\right) \in(0,+\infty)^{2}$ and the initial state $(\rho(0), z(0))=$ 
$\left(\left[K_{2} \rho_{0}+2 K_{1} \rho_{0}^{2}+0.5\right] /\left\{K_{1} \rho_{0}\right\}, 0.5\right)$ for (4), then the $z(t)$ component of the trajectory of (4) reaches 1 by time $t=$ $\rho_{0}$ and therefore leaves the state space. Hence, (3) cannot be used to give ISS or even global stability of the curve tracking dynamics (1). Instead, there are other curve tracking controllers that lead to nonlinear closed loop dynamics. For example, the control laws in [13] achieve obstacle avoidance and avoid possible singular configurations of the mobile robot, and also provide curve tracking. Even for systems in chained form, controllers with nonlinear closed loop dynamics are typically used [8].

Since the flow map of a nonlinear system cannot in general be given in closed form, one cannot prove ISS by directly constructing trajectories. Instead, it is convenient to use control analogs of Lyapunov's direct method, i.e., first construct a strict Lyapunov function for the closed loop dynamics, and then apply [3, Theorem 4.19]. However, the construction of the required global strict Lyapunov function for the curve tracking problem is challenging. While many Lyapunov functions have been built to establish asymptotic convergence of the closed loop tracking dynamics, to the best of our knowledge, they are all local [1] or non-strict. This does not pose a problem if the closed loop dynamics are linear, because in that case one can explicitly locate the poles of the linear system and prove exponential stability. On the other hand, for nonlinear dynamics, the existing convergence results rely on LaSalle Invariance Principle or variations of the Barbalat lemma, and so do not lend themselves to ISS.

In this note, we provide a method to construct a strict Lyapunov function for the curve tracking control law from [13] with nonlinear closed loop dynamics. The approach is a variant of the auxiliary function method from Matrosov theorems [6]. We found that applying the well known result [3, Theorem 4.19] on a sublevel set of a strict Lyapunov function establishes ISS with very small tolerance on the magnitude of the perturbations. To allow larger perturbations, we therefore developed a new ISS technique based on analyzing the forward invariance of a hexagonal region in the state space. This alternative method achieves ISS results for significantly larger perturbations on the control inputs and the states. We believe that our new work can be extended to other curve tracking controllers with nonlinear closed loop dynamics, and therefore allow more choices for robust curve tracking control laws in many other applications.

\section{Notation AND Definitions}

A continuous function $\gamma:[0,+\infty) \rightarrow[0,+\infty)$ belongs to class $\mathscr{K}$ (written $\gamma \in \mathscr{K}$ ) provided it is strictly increasing and $\gamma(0)=0$; if, in addition, $\gamma(r) \rightarrow+\infty$ as $r \rightarrow+\infty$, then it belongs to class $\mathscr{K}_{\infty}$ (written $\gamma \in \mathscr{K}_{\infty}$ ). A continuous function $\beta:[0,+\infty) \times[0,+\infty) \rightarrow[0,+\infty)$ is of class $\mathscr{K} \mathscr{L}$ (written $\beta \in \mathscr{K} \mathscr{L}$ ) provided (i) for each fixed $s \geq 0$, the function $\beta(\cdot, s)$ belongs to class $\mathscr{K}$, and (ii) for each fixed $r \geq 0$, the function $\beta(r, \cdot)$ is non-increasing and $\beta(r, s) \rightarrow 0$ as $s \rightarrow+\infty$. Let $\mathscr{U}$ be any subset of a Euclidean space such that $0 \in \mathscr{U}$ and $\mathscr{M}_{\mathscr{U}}$ denote the set of all measurable locally essentially bounded functions $\delta:[0,+\infty) \rightarrow \mathscr{U}$. For all $\delta \in \mathscr{M}_{\mathscr{U}}$ and real constants $a$ and $b$ with $b>a \geq 0$, let $|\delta|_{[a, b]}$ denote the essential supremum of the restriction of $\delta$ to $[a, b]$, and $|\delta|_{\infty}$ its essential supremum on $[0,+\infty)$.

Consider any open subset $\mathscr{X}$ of a Euclidean space and any point $\mathscr{E} \in \mathscr{X}$. A continuous function $\mathscr{V}: \mathscr{X} \rightarrow[0,+\infty)$ is positive semi-definite with respect to $\mathscr{E}$ provided $V(\mathscr{E})=0$; if, in addition, $\mathscr{V}(q)>0$ for all $q \in \mathscr{X} \backslash\{\mathscr{E}\}$, then $\mathscr{V}$ is positive definite with respect to $\mathscr{E}$. A function $\mathscr{V}$ is negative semi-definite (resp., definite) with respect to $\mathscr{E}$ provided $-\mathscr{V}$ is positive semi-definite (resp., definite) with respect to $\mathscr{E}$. Let $|p|_{\mathscr{E}}=|p-\mathscr{E}|$ denote the distance between any point $p \in \mathscr{X}$ and the point $\mathscr{E}$, where the distance is in the usual Euclidean metric. A function $\mathscr{V}: \mathscr{X} \rightarrow[0,+\infty)$ is a modulus with respect to $(\mathscr{E}, \mathscr{X})$ provided it is positive definite with respect to $\mathscr{E}$ and radially unbounded in the following sense: For each constant $M>0$, there is a constant $\delta_{M}>0$ (depending on $M$ ) such that $V(x) \geq M$ for all $x \in \mathscr{X}$ that satisfy either distance $(x$, boundary $(\mathscr{X})) \leq \delta_{M}$ or $|x|_{\mathscr{E}} \geq$ $1 / \delta_{M}$.

Consider a forward complete system $\dot{x}=\mathscr{F}(x, \delta)$ with state space $\mathscr{X}$ and disturbances $\delta \in \mathscr{M}_{\mathscr{U}}$, where $\mathscr{F}: \mathscr{X} \times$ $\mathscr{U} \rightarrow \mathscr{X}$ satisfies the standard existence and uniqueness of solutions properties for all initial conditions $x(0)=x_{0} \in \mathscr{X}$ and all disturbances $\delta \in \mathscr{M}_{\mathscr{U}}$, and $\mathscr{F}(\mathscr{E}, 0)=0$. Let $\mathscr{S} \subseteq \mathscr{X}$ be any neighborhood of $\mathscr{E}$. The system is input-to-state stable (ISS) with respect to $(\mathscr{U}, \mathscr{E}, \mathscr{S})$ provided there are functions $\beta \in \mathscr{K} \mathscr{L}$ and $\gamma \in \mathscr{K}_{\infty}$ such that $\left|x\left(t, x_{0}, \delta\right)\right|_{\mathscr{E}} \leq$ $\beta\left(\left|x_{0}\right|_{\mathscr{E}}, t\right)+\gamma\left(|\delta|_{[0, t]}\right)$ for all $t \geq 0$, all solutions $x\left(t, x_{0}, \delta\right)$ of the system with initial states $x_{0} \in \mathscr{S}$, and all $\delta \in \mathscr{M}_{\mathscr{U}}$. This agrees with the usual ISS condition when $\mathscr{X}=\mathscr{S}=\mathbb{R}^{n}$ and $\mathscr{E}=0$ [11]. A set $\mathscr{S}$ is forwardly invariant for the system with disturbances in $\mathscr{U}$ provided all trajectories with initial states in $\mathscr{S}$ remain in $\mathscr{S}$ for all positive times and all $\delta \in \mathscr{M}_{\mathscr{U}}$. The special case of ISS where $\mathscr{F}$ only depends on $x$ and the $\gamma$ term in the ISS estimate is not present is global asymptotic stability $(G A S)$ with respect to $(\mathscr{E}, \mathscr{S})$. In that case, a nonstrict (resp., strict) Lyapunov function for the system with respect to $(\mathscr{E}, \mathscr{X})$ is any $C^{1}$ modulus $V$ : $\mathscr{X} \rightarrow[0,+\infty)$ with respect to $(\mathscr{E}, \mathscr{X})$ such that the function $\dot{V}: \mathscr{X} \rightarrow \mathbb{R}$ defined by $\dot{V}(q)=\nabla V(q) \mathscr{F}(q)$ is negative semidefinite (resp., definite) with respect to $\mathscr{E}$. If $\dot{x}=\mathscr{F}(x)$ admits a strict Lyapunov function with respect to $(\mathscr{E}, \mathscr{X})$, then it is GAS with respect to $(\mathscr{E}, \mathscr{X})$.

\section{Feedback Design}

We next review the controller design from [13], which uses the Lyapunov function candidate

$$
V(\rho, \phi)=-\ln (\cos (\phi))+h(\rho),
$$

on $\mathscr{X}=(0,+\infty) \times(-\pi / 2, \pi / 2)$, where:

Assumption 1: The function $h:(0,+\infty) \rightarrow[0,+\infty)$ is $C^{1}, h^{\prime}$ has only finitely many zeros, $\lim _{\rho \rightarrow 0^{+}} h(\rho)=$ $\lim _{\rho \rightarrow+\infty} h(\rho)=+\infty$, and there is a constant $\rho_{0}>0$ such that $h\left(\rho_{0}\right)=0$.

The motivation for (5) is that the term $-\ln (\cos (\phi))$ penalizes the misalignment of the tangent vector to the curve at the closest point with the tangent vector of the moving 
vehicle, while the $h(\rho)$ term measures the separation between the moving vehicle and the obstacle. The time derivative of $V$ along all trajectories of (1) on the state space $\mathscr{X}=$ $(0,+\infty) \times(-\pi / 2, \pi / 2)$ is

$$
\begin{aligned}
\dot{V} & =\frac{\sin (\phi)}{\cos (\phi)} \dot{\phi}+h^{\prime}(\rho) \dot{\rho} \\
& =\frac{\sin (\phi)}{\cos (\phi)}\left[\frac{\kappa \cos (\phi)}{1+\kappa \rho}-u_{2}\right]-h^{\prime}(\rho) \sin (\phi) \\
& =\frac{\sin (\phi)}{\cos (\phi)}\left[\frac{\kappa \cos (\phi)}{1+\kappa \rho}-u_{2}-h^{\prime}(\rho) \cos (\phi)\right]
\end{aligned}
$$

Taking the controller

$$
u_{2}=\frac{\kappa \cos (\phi)}{1+\kappa \rho}-h^{\prime}(\rho) \cos (\phi)+\mu \sin (\phi)
$$

from [13] where $\mu>0$ is a steering constant gives

$$
\dot{V}=-\mu \frac{\sin ^{2}(\phi)}{\cos (\phi)} \leq 0 \text {. }
$$

This implies that $V$ is a nonstrict Lyapunov function for the corresponding closed-loop system

$$
\left\{\begin{array}{l}
\dot{\rho}=-\sin (\phi) \\
\dot{\phi}=h^{\prime}(\rho) \cos (\phi)-\mu \sin (\phi)
\end{array}\right.
$$

with respect to $(\mathscr{E}, \mathscr{X})$ for the state space $\mathscr{X}$ and the equilibrium $\mathscr{E}=\left(\rho_{0}, 0\right)$. Then [13] uses the LaSalle Invariance Principle to get global asymptotic stability properties for (8). However, robustness results are not considered in [13].

\section{Strict LyapunOV FUnCtion}

As we noted in the introduction, strict Lyapunov functions have the advantage that they can be used to certify important robustness features such as ISS. In this section, we transform the nonstrict Lyapunov function (5) into a strict Lyapunov function for (8), which will lead to our proof of ISS performance in Section VI. To define the transformation, we use the following additional condition:

Assumption 2: The $C^{2}$ function $h:(0,+\infty) \rightarrow[0,+\infty)$ satisfies Assumption 1 and the following three conditions:

(i) There is an increasing $C^{1}$ function $\gamma:[0,+\infty) \rightarrow[\mu,+\infty)$ such that for all $\rho>0$,

$$
\gamma(h(\rho)) \geq \max \left\{\mu, 1+0.5 \mu^{2}+h^{\prime \prime}(\rho)\right\} .
$$

(ii) There is a function $\Gamma \in \mathscr{K}_{\infty} \cap C^{1}$ such that for all $\rho>0$,

$$
\Gamma(h(\rho)) \geq h^{\prime}(\rho)^{2} .
$$

(iii) The function

$$
h^{\prime}(\rho)\left(\rho-\rho_{0}\right)
$$

is positive for all $\rho>0$ with $\rho \neq \rho_{0}$,

where $\rho_{0}>0$ is from Assumption 1 .

Remark 1: Assumption 2 is satisfied by

$$
h(\rho)=\alpha\left\{\rho+\frac{\rho_{0}^{2}}{\rho}-2 \rho_{0}\right\}
$$

for any constants $\alpha>0$ and $\rho_{0}>0$, if we take

$$
\gamma(q)=\frac{2}{\alpha^{2} \rho_{0}^{4}}\left(q+2 \alpha \rho_{0}\right)^{3}+1+0.5 \mu^{2}+\mu
$$

and $\Gamma(q)=4 q h^{\prime \prime}\left(\rho_{0}\right)+\bar{K} q^{4}$ for a large enough constant $\bar{K}>0$. In fact, since l'Hopital's Rule and (12) give $\lim _{\rho \rightarrow \rho_{0}}\left\{h^{\prime}(\rho)^{2} / h(\rho)\right\}=2 h^{\prime \prime}\left(\rho_{0}\right)>0$, we can find an open interval $\mathscr{I} \subseteq(0,+\infty)$ containing $\rho_{0}$ such that $\Gamma(h(\rho))$ $h^{\prime}(\rho)^{2}=h(\rho)\left[4 h^{\prime \prime}\left(\rho_{0}\right)+\bar{K} h(\rho)^{3}-\left\{h^{\prime}(\rho)^{2} / h(\rho)\right\}\right]>0$ for all $\rho \in \mathscr{I} \backslash\left\{\rho_{0}\right\}$ and any choice of $\bar{K}$. Then we can choose $\bar{K}$ such that $\bar{K} \geq h^{\prime}(\rho)^{2} / h(\rho)^{4}$ for all $\rho \in(0,+\infty) \backslash \mathscr{I}$, which gives (10).

Our strict Lyapunov function construction is:

Theorem 1: If Assumption 2 holds, then

$$
\begin{aligned}
U_{2}(\rho, \phi)= & -h^{\prime}(\rho) \sin (\phi)+\frac{1}{\mu} \int_{0}^{V(\rho, \phi)} \gamma(m) \mathrm{d} m \\
& +\Gamma(V(\rho, \phi))+V(\rho, \phi)
\end{aligned}
$$

is a strict Lyapunov function for $(8)$ with respect to $(\mathscr{E}, \mathscr{X})$ when $V$ is given by $(5)$ and $\mathscr{E}=\left(\rho_{0}, 0\right)$.

Proof: The proof is a variant of the Matrosov approach from [6, Chapter 5], using the auxiliary function $N(\rho, \phi)=-h^{\prime}(\rho) \sin (\phi)$, which is the Lie derivative of $h$ along all trajectories of (8). Along all trajectories of (8), we have $\dot{N}=-\left[h^{\prime}(\rho) \cos (\phi)\right]^{2}+h^{\prime}(\rho) \cos (\phi) \mu \sin (\phi)+$ $h^{\prime \prime}(\rho) \sin ^{2}(\phi)$. For all $(\rho, \phi) \in \mathscr{X}$, the triangle inequality gives $h^{\prime}(\rho) \cos (\phi) \mu \sin (\phi) \leq 0.5\left[h^{\prime}(\rho) \cos (\phi)\right]^{2}+$ $0.5 \mu^{2} \sin ^{2}(\phi)$, hence

$$
\begin{aligned}
\dot{N} \leq & -0.5\left[h^{\prime}(\rho) \cos (\phi)\right]^{2} \\
& +\left[0.5 \mu^{2}+h^{\prime \prime}(\rho)\right] \sin ^{2}(\phi) .
\end{aligned}
$$

From (9) and the fact that $\cos (\phi) \in(0,1]$ for all $\phi \in$ $(-\pi / 2, \pi / 2)$, we get

$$
\begin{aligned}
\dot{N} \leq & -0.5\left[h^{\prime}(\rho) \cos (\phi)\right]^{2}+\gamma(h(\rho)) \sin ^{2}(\phi) \\
& -\sin ^{2}(\phi) \\
\leq & -0.5\left[h^{\prime}(\rho) \cos (\phi)\right]^{2}-\sin ^{2}(\phi) \\
& +\gamma(V(\rho, \phi)) \frac{\sin ^{2}(\phi)}{\cos (\phi)} .
\end{aligned}
$$

Choose

$$
U_{1}(\rho, \phi)=N(\rho, \phi)+\frac{1}{\mu} \int_{0}^{V(\rho, \phi)} \gamma(m) \mathrm{d} m .
$$

By (7) and (16), we have

$$
\dot{U}_{1} \leq-0.5\left[h^{\prime}(\rho) \cos (\phi)\right]^{2}-\sin ^{2}(\phi) .
$$

The quantity $\alpha(\rho, \phi)=-0.5\left[h^{\prime}(\rho) \cos (\phi)\right]^{2}-\sin ^{2}(\phi)$ is negative definite with respect to $\mathscr{E}=\left(\rho_{0}, 0\right)$. However, $U_{1}$ is not necessarily a modulus with respect to $(\mathscr{E}, \mathscr{X})$.

On the other hand, we have $U_{2}=U_{1}+\Gamma(V)+V$, so (7) and (18) combine to give

$$
\begin{aligned}
& \dot{U}_{2} \leq-0.5\left[h^{\prime}(\rho) \cos (\phi)\right]^{2}-\sin ^{2}(\phi) \\
& -\left[\Gamma^{\prime}(V(\rho, \phi))+1\right] \mu \frac{\sin ^{2}(\phi)}{\cos (\phi)} \text {. }
\end{aligned}
$$

Therefore, $\dot{U}_{2}$ is negative definite with respect to $\mathscr{E}$ along all trajectories of (8). By (10), (14), and the inequality

$$
-h^{\prime}(\rho) \sin (\phi) \geq-h^{\prime}(\rho)^{2}-\frac{1}{4} \sin ^{2}(\phi),
$$

we deduce that

$$
\begin{aligned}
U_{2}(\rho, \phi) \geq \quad & -\frac{1}{4} \sin ^{2}(\phi)-\ln (\cos (\phi)) \\
& +\frac{1}{\mu} \int_{0}^{V(\rho, \phi)} \gamma(m) \mathrm{d} m
\end{aligned}
$$


Also, for all $\phi \in(-\pi / 2, \pi / 2)$, we have

$-\ln (\cos (\phi))=-0.5 \ln \left(1-\sin ^{2}(\phi)\right) \geq 0.5 \sin ^{2}(\phi)$.

Using (21)-(22) and the fact that $\gamma \geq \mu$ everywhere, we get

$$
\begin{aligned}
U_{2}(\rho, \phi) & \geq \frac{1}{4} \sin ^{2}(\phi)+\frac{1}{\mu} \int_{0}^{V(\rho, \phi)} \gamma(m) \mathrm{d} m \\
& \geq \frac{1}{\mu} \int_{0}^{V(\rho, \phi)} \gamma(m) \mathrm{d} m \\
& \geq V(\rho, \phi) .
\end{aligned}
$$

We conclude that $U_{2}$ is a modulus with respect to $(\mathscr{E}, \mathscr{X})$ for which $\dot{U}_{2}$ is negative definite with respect to $\mathscr{E}$ along all trajectories of (8). This proves the theorem.

\section{FORWARDLY INVARIANT HEXAGONS}

We saw in the introduction how the feedback linearization control law (3) produced a closed loop linear dynamics (4) that violated the necessary forward invariance condition of $\mathscr{X}=(0,+\infty) \times(-\pi / 2, \pi / 2)$. By contrast, we show next how the nonlinear controller (6) from [13] satisfies the required invariance conditions, even under perturbations of sufficiently small magnitude acting on the controller. In fact, a byproduct of our work is that for any compact subset $\mathscr{D} \subseteq \mathscr{X}$, we can build a closed hexagon $H$ in $\mathscr{X}$ such that for all trajectories $(\rho(t), \phi(t))$ for the closed loop perturbed system starting in $\mathscr{D}$, we have $(\rho(t), \phi(t)) \in H$ for all $t \geq 0$.

Therefore, consider the perturbed dynamics

$$
\left\{\begin{array}{l}
\dot{\rho}=-\sin (\phi) \\
\dot{\phi}=h^{\prime}(\rho) \cos (\phi)-\mu \sin (\phi)+\delta,
\end{array}\right.
$$

where the measurable essentially bounded functions $\delta$ represent actuator disturbances, the constant $\mu>0$ is to be chosen, and $h$ satisfies Assumption 2. To ensure that $\mathscr{X}=$ $(0,+\infty) \times(-\pi / 2, \pi / 2)$ is forwardly invariant for (24), we must restrict the magnitude of $\delta$. To see why, notice that if we choose $(\rho(0), \phi(0))=\left(2 \rho_{0}, 0\right)$ and $\delta \equiv \mu+\pi /\left(2 \rho_{0}\right)$, then we have $\rho(t) \geq \rho_{0}$ and so also $\phi(t) \geq \pi t /\left(2 \rho_{0}\right)$ for all $t \in\left[0, \rho_{0}\right]$, which gives $\phi\left(\rho_{0}\right) \geq \pi / 2$. From now on, we add the assumption that $h^{\prime \prime}(\rho)>0$ for all $\rho>0$; this holds for (12). For all constants $\rho_{*} \in\left(0, \rho_{0} / 3\right), \mu \in\left(0, \pi /\left(2 \rho_{*}\right)\right)$, and $K>2$, let $H\left(\rho_{*}, \mu, K\right)$ denote the hexagon in the $(\rho, \phi)$ plane having the vertices $A=\left(\rho_{*}, 0\right), B=\left(2 \rho_{*}, \mu \rho_{*}\right), C=$ $\left(\rho_{*}+K \rho_{0}, \mu \rho_{*}\right), D=\left(\rho_{*}+K \rho_{0}, 0\right), E=\left(K \rho_{0},-\mu \rho_{*}\right)$, and $F=\left(\rho_{*},-\mu \rho_{*}\right)$. Hence, $H\left(\rho_{*}, \mu, K\right)$ has a flat top and a flat bottom, and its other legs are vertical or have slope $\mu$.

For each compact subset $\mathscr{D} \subseteq \mathscr{X}$, we can choose $\rho_{*}, \mu$, and $K$ such that $\mathscr{D} \subseteq H\left(\rho_{*}, \mu, \bar{K}\right)$; see Fig. 1. Set

$$
\begin{aligned}
\Delta_{*} & =-h^{\prime}\left(3 \rho_{*}\right) \cos \left(\mu \rho_{*}\right), \\
\Delta_{* *} & =-\left|h^{\prime}\right|_{\left[\rho_{*}, \rho_{*}+K \rho_{0}\right]} \cos \left(\mu \rho_{*}\right)+\mu \sin \left(0.5 \mu \rho_{*}\right), \\
\Delta_{* * *} & =h^{\prime}\left(0.5 K \rho_{0}\right) \cos \left(\mu \rho_{*}\right), \text { and } \\
\delta_{*} & =\min \left\{\Delta_{*}, \Delta_{* *}, \Delta_{* * *}\right\} .
\end{aligned}
$$

For each $\rho_{*} \in\left(0, \rho_{0} / 3\right)$ and $K>2$, we can choose $\mu$ close enough to $\pi /\left(2 \rho_{*}\right)$ to ensure that $\Delta_{* *}>0$, because $-\left|h^{\prime}\right|_{\left[\rho_{*}, \rho_{*}+K \rho_{o}\right]} \cos \left(\mu \rho_{*}\right)$ is then close to 0 and $\mu \sin \left(0.5 \mu \rho_{*}\right)$ is close to $\mu / \sqrt{2}$. This gives $\delta_{*}>0$, since $h^{\prime}<0$ on $\left(0, \rho_{0}\right)$ and $h^{\prime}>0$ on $\left(\rho_{0},+\infty\right)$. We prove:

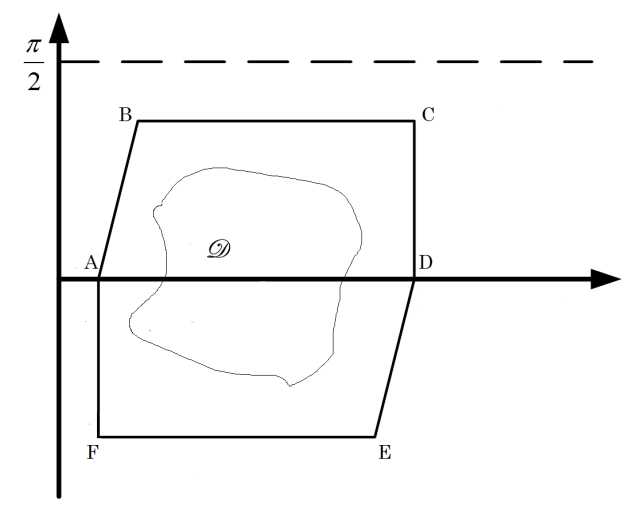

Fig. 1. Forwardly Invariant Hexagon $H\left(\rho_{*}, \mu, K\right)$ in $(\rho, \phi)$ Plane

Theorem 2: Let $\rho_{*} \in\left(0, \rho_{0} / 3\right), \mu \in\left(0, \pi /\left(2 \rho_{*}\right)\right)$, and $K>$ 2 be constants such that $\delta_{*}>0$. Then $H\left(\rho_{*}, \mu, K\right)$ is forwardly invariant for (24) with disturbances in $\mathscr{U}=\left[-\delta_{*}, \delta_{*}\right]$.

Proof: Our strategy is to prove that for all $\delta \in \mathscr{M}_{\mathscr{U}}$, the vector field $(\dot{\rho}, \dot{\phi})$ points into the hexagon at all points on the boundary of the hexagon, so no trajectory can leave the hexagon. Throughout the proof, we fix any $\delta \in \mathscr{M}_{\mathscr{U}}$. For each pair $(\rho, \phi) \in \mathscr{X}$, the function $\mathscr{I}: \mathscr{X} \rightarrow \mathbb{R}$ defined by $\mathscr{I}(\rho, \phi)=\phi-\mu \rho$ satisfies the following: For each $(\rho, \phi) \in$ $\mathscr{X}$, we have $y=\mu x+\mathscr{I}(\rho, \phi)$ when $(x, y)=(\rho, \phi)$. Hence, $\mathscr{I}(\rho, \phi)$ equals the $\phi$ axis intercept of the line that has the slope $\mu$ and passes through $(\rho, \phi)$. On the leg $A B$ of $H\left(\rho_{*}, \mu, K\right)$, we have $\rho \leq 2 \rho_{*}<\rho_{o}$, so $\dot{\mathscr{I}}=h^{\prime}(\rho) \cos (\phi)+$ $\delta \leq h^{\prime}\left(2 \rho_{*}\right) \cos (\phi)+\delta \leq h^{\prime}\left(2 \rho_{*}\right) \cos \left(\mu \rho_{*}\right)+\delta<0$ along all trajectories of (24), where the first two inequalities follow because $\cos (\phi)>0$ for all $\phi \in(-\pi / 2, \pi / 2), h^{\prime \prime}(\rho)>0$ for all $\rho>0$, and $h^{\prime}(\rho)<0$ on $\left(0, \rho_{o}\right)$; and the last inequality follows because $\delta_{*} \leq \Delta_{*}$ and because $h^{\prime \prime}(\rho)>0$ everywhere. Since $A B$ has slope $\mu$, it follows that no trajectory starting in $H\left(\rho_{*}, \mu, K\right)$ can exit $H\left(\rho_{*}, \mu, K\right)$ by passing through $A B$ except possibly through $A$ or $B$ (because if it did so at some time $t_{*}>0$, then for each constant $\varepsilon>0$, we could find a time $\tilde{t} \in\left(t_{*}, t_{*}+\varepsilon\right)$ such that $\mathscr{I}(\rho(\tilde{t}), \phi(\tilde{t}))>\mathscr{I}\left(\rho\left(t_{*}\right), \phi\left(t_{*}\right)\right)$, which is impossible, because $\dot{\mathscr{I}} \leq h^{\prime}\left(2 \rho_{*}\right) \cos \left(\mu \rho_{*}\right)+\delta_{*}<0$ at time $t_{*}$ and $\dot{\mathscr{I}}$ is continuous in the state). Also, $\dot{\rho}=$ $-\sin (\phi)>0$ all along the leg $A F$ except at $A$. At $A$, we have $\dot{\rho}=0$ and $\dot{\phi} \leq h^{\prime}\left(\rho_{*}\right)+\delta_{*}<0$, because the negativity of $h^{\prime}$ on $\left(0, \rho_{o}\right)$ and positivity of $h^{\prime \prime}$ imply that $\delta_{*} \leq \Delta_{*}<$ $-h^{\prime}\left(3 \rho_{*}\right)<-h^{\prime}\left(\rho_{*}\right)$. Hence, $\rho(t)>\rho_{*}$ and $\phi(t)<0$ for small enough times $t>0$ for all trajectories starting at $A$. Hence, no trajectory of (24) can exit $H\left(\rho_{*}, \mu, K\right)$ by passing through the left legs of $H\left(\rho_{*}, \mu, K\right)$, except possibly through $B$ or $F$. We will rule out exits from $B$ and $F$ when we consider the top and bottom legs.

Along the bottom $E F$, we get $\dot{\phi} \geq-\mu \sin \left(-\mu \rho_{*}\right)-$ $\left|h^{\prime}(\rho) \cos \left(-\mu \rho_{*}\right)\right|-|\delta| \geq-\left|h^{\prime}\right|_{\left[\rho_{*}, \rho_{*}+K \rho_{o}\right]} \cos \left(\mu \rho_{*}\right)+$ $\mu \sin \left(\mu \rho_{*}\right)-\delta_{*}>0$, since $\delta_{*} \leq \Delta_{* *}$. Similarly, along the top leg $B C$, we have $\dot{\phi} \leq-\mu \sin \left(\mu \rho_{*}\right)+\left|h^{\prime}(\rho) \cos \left(\mu \rho_{*}\right)\right|+|\delta| \leq$ $\left|h^{\prime}\right|_{\left[2 \rho_{*}, \rho_{*}+K \rho_{o}\right]} \cos \left(\mu \rho_{*}\right)-\mu \sin \left(\mu \rho_{*}\right)+\delta_{*}<0$, because $\left|h^{\prime}\right|_{\left[\rho_{*}, K \rho_{o}+\rho_{*}\right]} \geq\left|h^{\prime}\right|_{\left[2 \rho_{*}, K \rho_{o}+\rho_{*}\right]}$, which follows because $h^{\prime}<0<h^{\prime \prime}$ on $\left(0,2 \rho_{*}\right)$. Combined with the preceding paragraph, we conclude that no trajectory starting in $H\left(\rho_{*}, \mu, K\right)$ can exit through the left legs, top, or bottom of 
$H\left(\rho_{*}, \mu, K\right)$, except possibly through $C$ or $E$. We next rule out exits from $C$ and $E$ by considering the right legs of the hexagon. Along $C D$, we have $\dot{\rho}<0$, except at $D$. At $D$, we have $\dot{\rho}=0$ and $\dot{\phi}=h^{\prime}\left(\rho_{*}+K \rho_{o}\right)+\delta>h^{\prime}\left(0.5 K \rho_{o}\right)-\delta_{*}>0$, because $h^{\prime \prime}>0$ everywhere and $\delta_{*} \leq \Delta_{* * *}$. It follows that $\rho(t)<\rho_{*}+K \rho_{o}$ and $\phi(t)>0$ hold for small enough times $t>0$ along trajectories starting at $D$. Moreover, $\dot{\mathscr{I}} \geq h^{\prime}\left(K \rho_{o}\right) \cos \left(\mu \rho_{*}\right)+\delta>0$ along $D E$, because $\delta_{*} \leq \Delta_{* * *}$. Hence, arguing as we did for $A B$, we conclude that no trajectory can exit $H\left(\rho_{*}, \mu, K\right)$ through the right legs. This proves the result.

\section{InPUT-TO-STATE STABILITy}

We next combine our strict Lyapunov function and invariant hexagon constructions to prove:

Theorem 3: Let $h$ satisfy Assumption 2 and $\mathscr{D} \subseteq \mathscr{X}$ be any compact neighborhood of $\left(\rho_{0}, 0\right)$. Assume that $h^{\prime \prime}(\rho)>$ 0 for all $\rho>0$. Choose any constants $\rho_{*} \in\left(0, \rho_{0} / 3\right), \mu \in$ $\left(0, \pi /\left(2 \rho_{*}\right)\right)$, and $K>2$ such that $\mathscr{D} \subseteq H\left(\rho_{*}, \mu, K\right)$ and $\delta_{*}>$ 0 . Then we can find constants $\bar{\gamma}_{i}>0$ (depending on $\rho_{*}, \mu$, and $K$ ) such that

$$
|(\rho(t), \phi(t))|_{\mathscr{E}} \leq \bar{\gamma}_{1} e^{-\bar{\gamma}_{2} t}|(\rho(0), \phi(0))|_{\mathscr{E}}+\bar{\gamma}_{3}|\delta|_{[0, t]}
$$

for all perturbations $\delta \in \mathscr{M}_{\left[-\delta_{*}, \delta_{*}\right]}$, all trajectories $(\rho, \phi)$ : $[0,+\infty) \rightarrow \mathbb{R}^{2}$ of (24) starting in $\mathscr{D}$, and all $t \geq 0$. In particular, (24) is ISS with respect to $(\mathscr{U}, \mathscr{E}, \mathscr{D})$ for the equilibrium $\mathscr{E}=\left(\rho_{0}, 0\right)$ and the disturbance set $\mathscr{U}=\left[-\boldsymbol{\delta}_{*}, \boldsymbol{\delta}_{*}\right]$.

Proof: In what follows, all (in)equalities should be understood to hold globally unless otherwise indicated, and we only consider trajectories that start in $\mathscr{D}$, and therefore remain in $H\left(\rho_{*}, \mu, K\right)$ by Theorem 2 . Along all trajectories of (24) with $\delta \in \mathscr{M}_{\left[-\delta_{*}, \delta_{*}\right]}$, (19) gives

$$
\begin{aligned}
\dot{U}_{2} \leq & -0.5\left[h^{\prime}(\rho) \cos (\phi)\right]^{2}-\sin ^{2}(\phi) \\
& -\left[\Gamma^{\prime}(V(\rho, \phi))+1\right] \mu \frac{\sin ^{2}(\phi)}{\cos (\phi)}+\frac{\partial U_{2}}{\partial \phi}(\rho, \phi) \delta(t) .
\end{aligned}
$$

Recalling the formula (14) for $U_{2}$ gives

$$
\begin{aligned}
\frac{\partial U_{2}}{\partial \phi}(\rho, \phi)= & -h^{\prime}(\rho) \cos (\phi)+\left\{\frac{1}{\mu} \gamma(V(\rho, \phi))\right. \\
& \left.+\Gamma^{\prime}(V(\rho, \phi))+1\right\} \frac{\sin (\phi)}{\cos (\phi)} .
\end{aligned}
$$

Combining (27)-(28) gives

$$
\begin{aligned}
\dot{U}_{2} \leq & -0.5\left[h^{\prime}(\rho) \cos (\phi)\right]^{2}-\sin ^{2}(\phi) \\
& -\left[\Gamma^{\prime}(V(\rho, \phi))+1\right] \mu \frac{\sin ^{2}(\phi)}{\cos (\phi)} \\
+ & {\left[\left|h^{\prime}(\rho) \cos (\phi)\right|+\left\{\frac{1}{\mu} \gamma(V(\rho, \phi))\right.\right.} \\
& \left.\left.+\Gamma^{\prime}(V(\rho, \phi))+1\right\}\left|\frac{\sin (\phi)}{\cos (\phi)}\right|\right]|\delta(t)| .
\end{aligned}
$$

Since $\left|h^{\prime}(\rho) \cos (\phi)\right||\delta(t)| \leq \frac{1}{4}\left[h^{\prime}(\rho) \cos (\phi)\right]^{2}+\delta(t)^{2}$, we get

$$
\begin{aligned}
\dot{U}_{2} \leq & -\frac{1}{4}\left[h^{\prime}(\rho) \cos (\phi)\right]^{2}-\sin ^{2}(\phi) \\
& -\left[\Gamma^{\prime}(V(\rho, \phi))+1\right] \mu \frac{\sin ^{2}(\phi)}{\cos (\phi)} \\
& +\left\{\frac{1}{\mu} \gamma(V(\rho, \phi))\right. \\
& \left.+\Gamma^{\prime}(V(\rho, \phi))+1\right\}\left|\frac{\sin (\phi)}{\cos (\phi)}\right||\delta(t)|+\delta(t)^{2} .
\end{aligned}
$$

Also, $\lim _{\rho \rightarrow \rho_{0}} h(\rho) / h^{\prime}(\rho)^{2}=\lim _{\rho \rightarrow \rho_{0}} 1 /\left\{2 h^{\prime \prime}(\rho)\right\}>0$ and $\lim _{\phi \rightarrow 0}\left[-\ln (\cos (\phi)) / \sin ^{2}(\phi)\right]=0.5$, by l'Hopital's Rule. Hence, can find a constant $\bar{c}$ such that $-\ln (\cos (\phi)) \leq$ $\bar{c} \sin ^{2}(\phi)$ and $h(\rho) \leq \bar{c} h^{\prime}(\rho)^{2}$ hold for all $(\rho, \phi) \in$ $H\left(\rho_{*}, \mu, K\right)$. Set $J=\max \left\{V(\rho, \phi):(\rho, \phi) \in H\left(\rho_{*}, \mu, K\right)\right\}$ and $\bar{c}_{1}=1+\{\gamma(J) / \mu\}+\max \left\{\Gamma^{\prime}(r): 0 \leq r \leq J\right\}$. Then (14) gives

$$
\begin{aligned}
U_{2}(\rho, \phi) \leq & \left\{\left|h^{\prime}(\rho)\right|\right\}\{|\sin (\phi)|\}+\bar{c}_{1} V(\rho, \phi) \\
\leq & 0.5 h^{\prime}(\rho)^{2}+0.5 \sin ^{2}(\phi) \\
& +\bar{c}_{1}[h(\rho)-\ln (\cos (\phi))] \\
\leq & {\left.\left[0.5+\bar{c} \bar{c}_{1}\right]\left[h^{\prime}(\rho)^{2}+\sin ^{2}(\phi)\right)\right] } \\
\leq & \left.\frac{\left[0.5+\bar{c} \bar{c}_{1}\right]}{\cos ^{2}\left(\mu \rho_{*}\right)}\left[\left(h^{\prime}(\rho) \cos (\phi)\right)^{2}+\sin ^{2}(\phi)\right)\right]
\end{aligned}
$$

and

$$
\begin{aligned}
& {\left[\frac{1}{\mu} \gamma(V(\rho, \phi))+\Gamma^{\prime}(V(\rho, \phi))+1\right]\left|\frac{\sin (\phi)}{\cos (\phi)}\right||\delta|} \\
& \leq\left\{\sqrt{\mu} \frac{|\sin (\phi)|}{\sqrt{\cos (\phi)}}\right\}\left\{\frac{\bar{c}_{1}|\delta|}{\sqrt{\mu \cos (\phi)}}\right\} \leq \frac{\mu \sin ^{2}(\phi)}{2 \cos (\phi)}+\frac{\bar{c}_{1}^{2}}{2 \mu \cos \left(\mu \rho_{*}\right)} \delta^{2}
\end{aligned}
$$

hold for all $\delta \in \mathbb{R}$ and all $(\rho, \phi) \in H\left(\rho_{*}, \mu, K\right)$, by applying the triangle inequality $a b \leq \frac{1}{2} a^{2}+\frac{1}{2} b^{2}$ to the terms in braces. Therefore, our decay estimate (30) on $U_{2}$ gives $\dot{U}_{2} \leq-\bar{c}_{2} U_{2}+\bar{c}_{3}|\delta|_{\infty}^{2}$ along all trajectories of (24) in the hexagon, where $\bar{c}_{2}=0.25 \cos ^{2}\left(\mu \rho_{*}\right) /\left\{0.5+\bar{c} \bar{c}_{1}\right\}$ and $\bar{c}_{3}=$ $1+\bar{c}_{1}^{2} /\left\{2 \mu \cos \left(\mu \rho_{*}\right)\right\}$. Applying Variation of Parameters now gives $U_{2}(\rho(t), \phi(t)) \leq e^{-\bar{c}_{2} t} U_{2}(\rho(0), \phi(0))+\bar{c}_{3}|\delta|_{\infty}^{2} / \bar{c}_{2}$ along all trajectories of (24) in the hexagon for which $\delta \in$ $\mathscr{M}_{\left[-\delta_{*}, \delta_{*}\right]}$. Using (23) and (31), we can find positive constants $\bar{c}_{4}$ and $\bar{c}_{5}$ such that

$$
\bar{c}_{4}|(\rho, \phi)|_{\mathscr{E}}^{2} \leq V(\rho, \phi) \leq U_{2}(\rho, \phi) \leq \bar{c}_{5}|(\rho, \phi)|_{\mathscr{E}}^{2}
$$

for all $(\rho, \phi) \in H\left(\rho_{*}, \mu, K\right)$. Since causality implies that the trajectory at time $t$ does not depend on values of the disturbance at later times, we therefore get the ISS estimate (26) with the choices $\bar{\gamma}_{1}=\sqrt{\bar{c}_{5} / \bar{c}_{4}}, \bar{\gamma}_{2}=0.5 \bar{c}_{2}$, and $\bar{\gamma}_{3}=$ $\sqrt{\bar{c}_{3} /\left\{\bar{c}_{2} \bar{c}_{4}\right\}}$, by applying the general relation $\sqrt{p+q} \leq$ $\sqrt{p}+\sqrt{q}$, which holds for all $p \geq 0$ and $q \geq 0$.

\section{Simulations}

To demonstrate our ISS results, we simulated the perturbed curve tracking dynamics (24) with the choice

$$
h(\rho)=10\left\{\rho+\frac{1}{\rho}-2\right\}
$$

for $h$. This is the special case of (12) with $\alpha=10$ and $\rho_{0}=1$. Therefore, it satisfies our Assumption 2 on $h$; see Fig. 2. We took $\rho_{*}=0.22, \mu=6.42$ and $K=20$, to get the compact invariant hexagon $H(0.22,6.42,20) \subseteq \mathscr{X}$. Using the notation from (25) gives $\Delta_{*}=-h^{\prime}\left(3 \rho_{*}\right) \cos \left(\mu \rho_{*}\right)=2.03$, $\Delta_{* *}=-\left|h^{\prime}\right|_{\left[\rho_{*}, \rho_{*}+K \rho_{0}\right]} \cos \left(\mu \rho_{*}\right)+\mu \sin \left(0.5 \mu \rho_{*}\right)=2.612$, and $\Delta_{* * *}=h^{\prime}\left(0.5 K \rho_{0}\right) \cos \left(\mu \rho_{*}\right)=1.55$. Hence, our bound on $\delta$ to maintain forward invariance of $H(0.22,6.42,20)$ for (24) is $\delta_{*}=\min \left\{\Delta_{*}, \Delta_{* *}, \Delta_{* * *}\right\}=1.55$.

We obtained two simulations for (24). First we took the initial state $(1,1.4) \in H(0.22,6.42,20)$ and the zero disturbance $\delta \equiv 0$. In Figs. 3-4, we plot the corresponding trajectories for $\rho(t)$ and $\phi(t)$, which show convergence toward the 
equilibrium $\mathscr{E}=(1,0)$. Our second simulation used the same data as our first, except we took the disturbance $\delta \equiv 1.55$. With the added disturbance, the convergence $\phi(t) \rightarrow 0$ is maintained, but we no longer have $\rho(t) \rightarrow \rho_{0}=1$; see Figs. 5-6. This illustrates the effect of the perturbation through the overflow term in the ISS estimate.

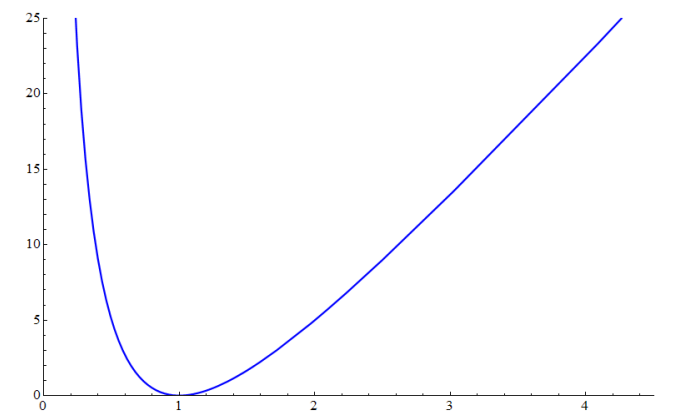

Fig. 2. Choice of $h\left(\rho^{2}\right)=10\left(\rho+3^{3}\{1 / \rho\}-2\right)^{4}$ from (33)

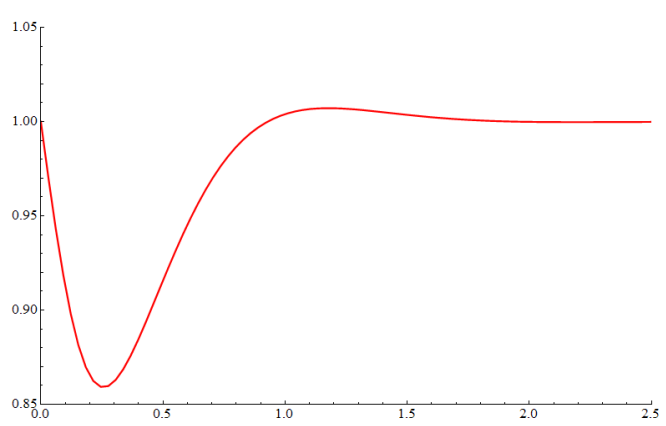

Fig. 3. $\rho(t)$ with Perturbation $\delta \equiv 0$

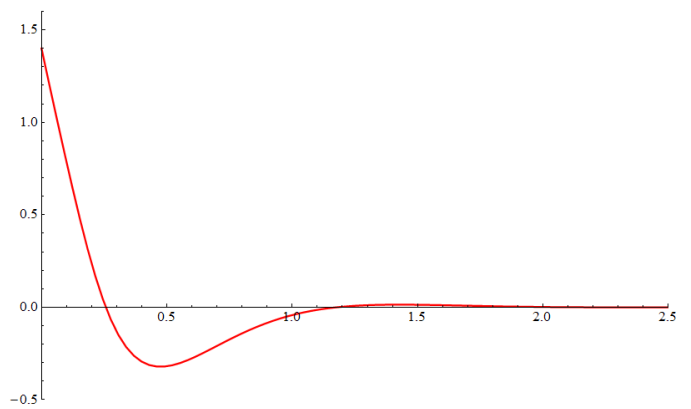

Fig. 4. $\phi(t)$ with Perturbation $\delta \equiv 0$

VIII. CONCLUSIONS

Curve tracking is a fundamental behavior in robotics. While the two dimensional tracking dynamics are feedback linearizable, the linear system does not satisfy the necessary invariance properties. Although Lyapunov functions have been used for curve tracking, to the best of our knowledge, the earlier Lyapunov functions are all nonstrict and so do not lend themselves to ISS performance analysis. Our strict Lyapunov function construction enabled us to use ISS to quantify the robust performance of the controller design from [13]. It would be useful to extend our analysis to three dimensional curve tracking problems and feedback delays.

\section{REFERENCES}

[1] Z-P. Jiang and H. Nijmeijer. "Tracking control of mobile robots: A case study in backstepping," Automatica, Volume 33, Number 7, pp. 1393-1399, 1997.

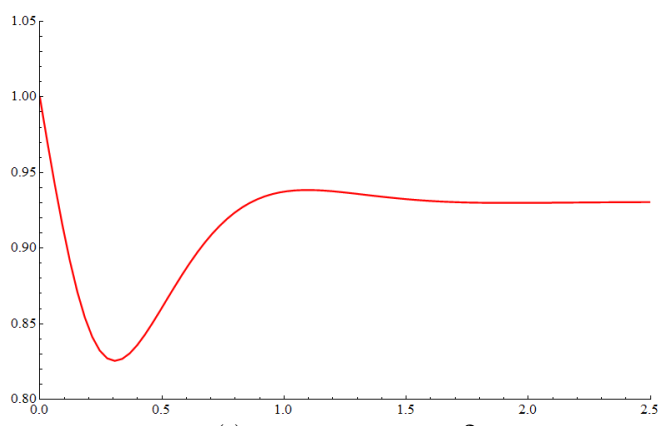

Fig. 5. $\rho(t)$ with Perturbation $\delta \equiv 1.55$

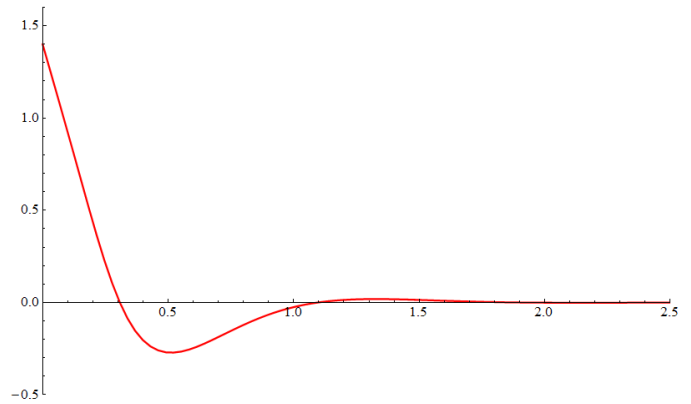

Fig. 6. $\phi(t)$ with Perturbation $\delta \equiv 1.55$

[2] E. Justh and P. Krishnaprasad, "Natural frames and interacting particles in three dimensions," in Proceedings of the 44th IEEE Conference on Decision and Control and European Control Conference (Seville, Spain, December 2005), pp. 2841-2846.

[3] H. Khalil, Nonlinear Systems, Third Edition, Prentice Hall, Upper Saddle River, NJ, 2002.

[4] R. Lenain, B. Thuilot, C. Cariou, and P. Martinet, "High accuracy path tracking for vehicles in presence of sliding: Application to farm vehicle automatic guidance for agricultural tasks," Autonomous Robots, Volume 21, Number 1, pp. 79-97, 2006.

[5] V. Lumelsky and A. Stepanov, "Path planning strategies for a point mobile automaton moving amidst unknown obstacles of arbitrary shape," Algorithmica, Volume 2, Number 2, pp. 403-430, 1987.

[6] M. Malisoff and F. Mazenc, Constructions of Strict Lyapunov Functions, Communications and Control Engineering Series, SpringerLondon Ltd., London, UK, 2009.

[7] A. Micaelli and C. Samson, "Trajectory tracking for unicycle-type and two-steering-wheels mobile robots," INRIA Report 2097, 1993.

[8] P. Morin and C. Samson, "Motion control of wheeled mobile robots," in B. Siciliano and O. Khatib, editors, Springer Handbook of Robotics, pages 799-826. Springer-Verlag, Berlin, Germany, 2008.

[9] C. Samson, "Control of chained systems: Application to pathfollowing and time-varying point-stabilization of mobile robots," IEEE Transactions on Automatic Control, Volume 40, Number 1, pp. 64-77, 1995.

[10] E.D. Sontag, "Stabilizability, i/o stability and coprime factorizations," in Proceedings of the IEEE Conference on Decision and Control (Austin,TX, December 1988), pp. 457-458.

[11] E.D. Sontag, "Input-to-state stability: Basic concepts and results," in P. Nistri and G. Stefani, editors, Nonlinear and Optimal Control Theory. Springer, Berlin, Germany, 2008.

[12] F. Zhang, D.M. Fratantoni, D. Paley, J. Lund, and N. Leonard, "Control of coordinated patterns for ocean sampling," International Journal of Control, Volume 80, Number 7, pp. 1186-1199, 2007.

[13] F. Zhang, E. Justh, and P. Krishnaprasad, "Boundary following using gyroscopic control," in Proceedings of the 43rd IEEE Conference on Decision and Control (Paradise Island, Bahamas, December 2004), pp. 5204-5209.

[14] F. Zhang and N. Leonard, "Coordinated patterns of unit speed particles on a closed curve," Systems and Control Letters, Volume 56, Number 6, pp. 397-407, 2007.

[15] F. Zhang, A. O'Connor, D. Luebke, and P. Krishnaprasad, "Experimental study of curvature-based control laws for obstacle avoidance," in Proceedings of IEEE International Conference on Robotics and Automation (New Orleans, LA, April-May 2004), pp. 3849-3854. 